\title{
Ultrasound Guided Erector Spinae Plane Block versus Thoracic Epidural for Post-mastectomy Analgesia
}

\author{
Ismail M. A. Ahmed ${ }^{1 *}$ MD and Hesham S. Abdelraouf ${ }^{1}$ MD
}

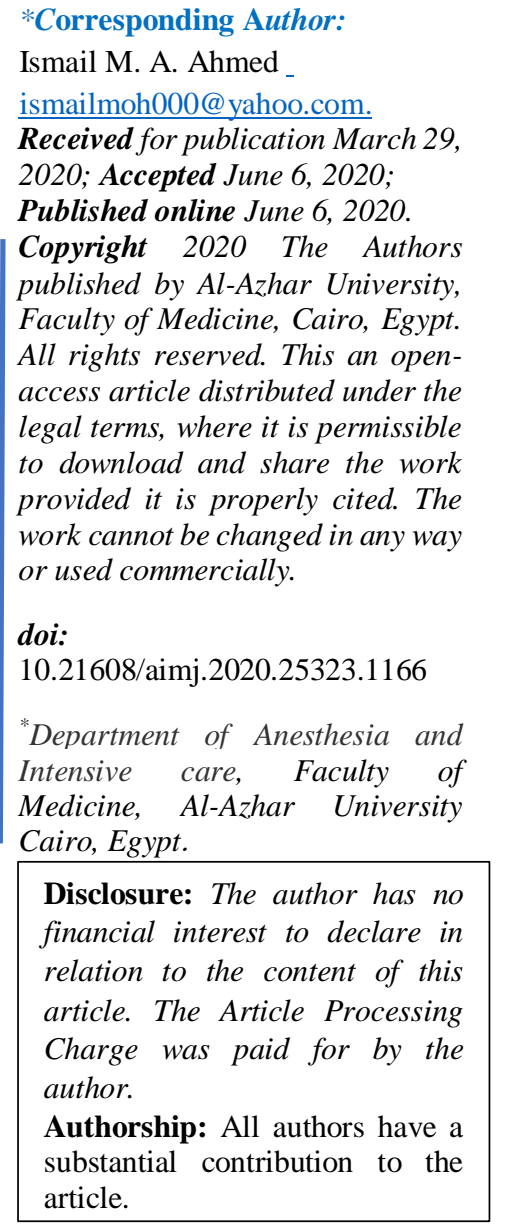

\begin{abstract}
Background: Thoracic epidural analgesia (TEA) was considered as the gold standard for postoperative analgesia in thoracic surgeries, particularly in bilateral procedures. However, it isn't routinely used as it is associated with some haemodynamic side effects. Erector spinae plane (ESP) block is recognized as a promising postoperative analgesia technique.

Aim of the study: The primary outcome was to compare post-mastectomy pain control in TEA and ESP block groups, and secondary outcomes were to compare post-operative hypotension, number of morphine boluses, and patient satisfaction.

Patient and Methods: Sixty female patients scheduled for elective mastectomy were enrolled in this study. Patients were allocated into 2 groups, 30 patients each. Group (TE) patients underwent ultrasoundguided single-shot TEA, while group (ES) were handled with ultrasoundguided single-shot ESP block. Peri-operative details, Post-operative hypotension, visual analog scale (VAS) assessment of pain, number of postoperative morphine boluses, and patient satisfaction were recorded.

Results: Time needed to give block was shorter in the group (ES) $(\mathrm{p}<0.001)$. Group (ES) patients reported significantly lower pain scores according to VAS $(\mathrm{P}<0.001)$. Hypotension was more recorded in group (TE) patients, with statistical significance at 0 point $(\mathrm{p}<0.001)$. Required post-operative morphine boluses were significantly lower in the group (ES), as $3.3 \%$ of patients received 3 boluses compared to $27 \%$ in the group (TE) $(\mathrm{p}=0.01)$. More patients were highly satisfied with group ES $(60 \%)$ than group TE $(30 \%)(\mathrm{p}=0.037)$.

Conclusion: Ultrasound-guided ESP block is a choice with a better outcome in patients undergoing elective mastectomy as regards the lower frequency of hypotension, better efficiency, and patient satisfaction.

Keywords: Ultrasound-guided Erector Spinae Plane block; pain; postmastectomy.
\end{abstract}

\section{INTRODUCTION}

Breast surgery is a common procedure, particularly in middle-aged women ${ }^{1,2}$. The procedure is associated with an increased incidence of acute and chronic pain $^{2}$. Postoperative analgesia following breast surgery extending beyond lumpectomy is sometimes challenging, about $20 \%$ to $50 \%$ were recorded to develop post-mastectomy pain syndrome ${ }^{3}$. Multimodal techniques for pain management have been recommended by The American Society of Anesthesiologists (ASA) task force for the management for acute postoperative pain ${ }^{4,5}$. These techniques include regional analgesia, intravenous (IV), and oral analgesics as opioids, paracetamol, and nonsteroidal anti-inflammatory drugs (NSAIDs) ${ }^{5}$.
Inappropriate postoperative analgesia may increase morbidity and mortality ${ }^{6}$. Satisfactory postoperative analgesia prevents unnecessary patient discomfort, may play a role in decreasing morbidity, postoperative hospital stay, and the cost ${ }^{7}$. When opioids are used solely for analgesia, they may cause nausea, vomiting, pruritus, and respiratory depression. Regional techniques, especially Thoracic Epidural Analgesia (TEA) have been described to reduce the postoperative pain with improved outcome 5,8 .

Ultrasound-guided (USG) erector spinae plane (ESP) block has become a recognizable technique for regional analgesia in thoracic surgeries ${ }^{9}$. This block can be given unilaterally for simple mastectomy surgery. A local anaesthetic (LA) is dripped deep to the erector spinae muscle and superficial to the tip of 
the transverse process at the myofascial plane. The instilled LA can induce sensory block at the multidermatomal levels across the posterior, lateral, and anterior thoracic wall, probably due to the diffusion into the paravertebral space. In addition to its effect at the rami communicans that supply the sympathetic chain ${ }^{10}$, the ESP block affects the dorsal and ventral primary rami of the thoracic nerves ${ }^{11}$. ESP is relatively easier to apply in breast surgeries as compared to neuraxial, nerve blocks, TEA, and other regional modalities $5,9,12$.

\section{Aim of the work}

The primary outcome was to compare postmastectomy pain control in TEA and ESP block groups, and the secondary outcomes were to compare the post-operative hypotension, the number of morphine boluses, and patient satisfaction.

\section{PATIENT AND METHODS}

This prospective randomized clinical study was conducted on sixty female patients, aged 40-60-yearold, with the American Society of Anesthesiologists' physical status I-II enrolled for elective simple mastectomy. The study was done between March and October 2019 in a military tertiary care center in Saudi Arabia after approval of the local ethics committee and informed written consent. Randomization was performed to two groups each of 30 , using computergenerated closed and opaque envelopes method to 2 groups according to the technique used; ultrasoundguided single-shot Thoracic Epidural Analgesia (TE) group, and ultrasound-guided single shot Erector Spinae Plane block (ES) group.

All patients who completed the study had a clinical assessment on the pre-operative visit.

Exclusion criteria

Patient refusal, Morbidly obese (BMI $\geq 40$ ), Anomalies of the vertebral column, patient on anti-coagulants, bleeding diathesis.

In both groups, the procedures were done under the strict aseptic precautions, at the level of thoracic intervertebral space 4/5 (T4/T5). A high-frequency 12 $\mathrm{MHz}$ linear ultrasound transducer (Philips En Visor CHD, Bothell, Washington, USA 98041) was used as real-time scanning in ESP group, and as pre-insertion scanning and assessment in TEA group.

Technique

On arrival at the operating theatre, patients were connected to standard monitors; non-invasive arterial blood pressure (NIBP), electrocardiogram (ECG), and pulse oximetry ( $\mathrm{SpO} 2)$. Balanced general anesthesia (GA) was induced with propofol $2 \mathrm{mg} / \mathrm{kg}$, fentanyl 1 $\mu \mathrm{g} / \mathrm{kg}$, rocuronium $0.6 \mathrm{mg} / \mathrm{kg}$, and tracheal intubation was done in both groups. Anesthesia was maintained with sevoflurane $1.8 \%$ and $50 \%$ nitrous oxide in oxygen.

After the end of surgery and before extubation, the studied technique was done.

In Group TE (TEA),

A linear transducer was placed in a horizontal orientation at the $\mathrm{T} 5$ spinous process corresponding to T4 transverse process. Three muscles trapezius (uppermost), rhomboids major (middle), and erector spinae (lowermost) were identified superior to the hyperechoic transverse process. Site of insertion, skin to epidural space distance, and direction were identified and marked before insertion of an 18 Gauge Touhi needle (prefix epidural Catheter set, b Braun) (Figure 1), then $20 \mathrm{ml}$ BUPIVACAINE $0.25 \%$ (0.25\% BUPIVACAINE $\mathrm{HCl}$ inj, USP HOSPIRA INC USA) was injected after negative aspiration. In Group ES (ESP Block),

A linear transducer was placed in a longitudinal orientation $3 \mathrm{~cm}$ lateral to the T4 spinous process. Three muscles trapezius (uppermost), rhomboids major (middle), and erector spinae (lowermost) were identified superior to the hyperechoic transverse process (Figure 2). Using in-plane approach an 22 Gauge echogenic needle (sonolex STIM PAJUNK) was inserted in caudal-cephalad direction, until the tip is deep to erector spinae muscle, as evidenced by visible hydro dissection below the muscle plane, then $20 \mathrm{ml}$ bupivacaine $0.25 \%$ was injected after negative aspiration.

Time to give the block was assessed in both groups in minutes. Extubation was done after satisfying extubation criteria, then patients were transferred to the recovery room.

The pain assessment after full recovery was performed using a $10 \mathrm{~cm}$ visual analog scale (VAS) (0- no pain and $10 \mathrm{~cm}$ maximum pain) ${ }^{13}$. The postoperative pain assessments using VAS at rest and during cough were performed postoperatively at 0 point (the full recovery state), $1 \mathrm{hr}, 2 \mathrm{hr}, 4 \mathrm{hr}, 6 \mathrm{hr}$, and at $24 \mathrm{hr}$. IV paracetamol $1 \mathrm{~g}$ every 6 hours was administered. Rescue analgesia was administered, if VAS was $\geq 4$ at rest or on patient's demand, with IV morphine sulphate $0.03 \mathrm{mg} / \mathrm{kg}$. the number of morphine boluses and the total amount in the first postoperative 24 hours was calculated. Mean arterial blood pressure (MABP) was assessed postoperatively at 0 point (immediately after the procedure), $1 \mathrm{hr}, 2 \mathrm{hr}, 4 \mathrm{hr}, 6 \mathrm{hr}$, and at $24 \mathrm{hr}$. Patient satisfaction related to block performance, postoperative pain relief was evaluated by an 11- point satisfaction score $(0=$ unsatisfied and $10=$ most satisfied) ${ }^{14}$, and the score was divided as follows 0-3 (not satisfied), 4-6 (partly satisfied), and 7-10 (highly satisfied).
Anesthesia and

Intensive care 


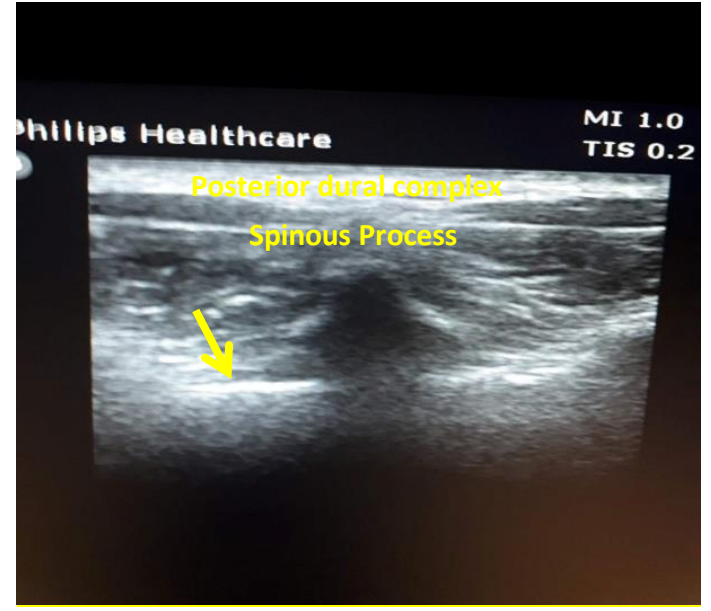

Figure (1): Ultrasound view. Thoracic Epidural.

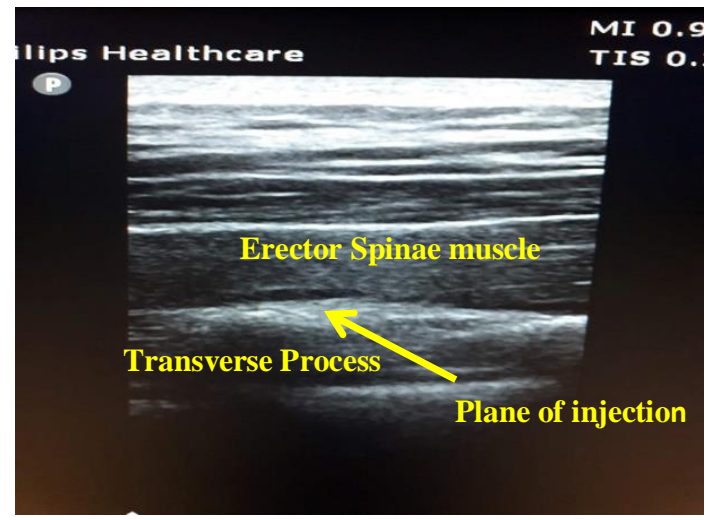

Figure (2): ultrasound view. Erector Spinae Plane.

\section{Statistical analysis}

A pilot study was conducted on 20 females indicated for elective mastectomy and post hoc analysis was performed using VAS scores with an alpha error (Type I) of 0.05 and calculated the beta error (Type
II) $90 \%$. A total of 30 patients in each group completed the study; Group TE and Group ES.

Data were fed to the computer and analyzed using IBM SPSS software package version 20.0. (Armonk, NY: IBM Corp). The Kolmogorov- Smirnov test was used to verify the normality of distribution of variables, Comparisons between groups for categorical variables were assessed using Chi-square test (Fisher or Monte Carlo). The student $t$-test was used to compare two groups for normally distributed quantitative variables. Mann Whitney test was used to compare between two groups for non-normally distributed quantitative variables. P-value was considered significant at $<0.05$.

\section{RESULTS}

The demographic data (age, weight), ASA status were comparable between the studied groups $(P>0.05)$ as shown in Table (1). Surgery duration was comparable between the 2 groups, while time to give the block was significantly lower in group ES as shown in Table (2). Post-operative MABP readings were lower in group TE of patients with a statistically significant decrease at 0 point $(\mathrm{p}<0.001)$ as shown in table (3).As regards post-operative pain using VAS score, there was statistically significant decrease in group (ES) patients $(\mathrm{P}<0.001)$ as shown in table $(4)$. The required post-operative morphine boluses were significantly lower in the group (ES) than group (TE) patients, as $3.3 \%$ of patients in $2^{\text {nd }}$ group received 3 boluses compared to $27 \%$ of the first group $(\mathrm{p}=0.01)$ as shown in table (5). As regards patient satisfaction, more patients were highly satisfied in this study in group ES $(60 \%)$ than group TE $(30 \%)(\mathrm{p}=0.037)$ as shown in table (6).
Anesthesia and Intensive care

\begin{tabular}{cccc}
\hline & $\begin{array}{c}\text { Group TE } \\
(\mathbf{n = 3 0})\end{array}$ & $\begin{array}{c}\text { Group ES } \\
(\mathbf{n = 3 0})\end{array}$ & P-value \\
\hline Age (years) & $50.5 \pm 5.3$ & $51.2 \pm 4.9$ & 0.596 \\
BMI & $29.48 \pm 2.36$ & $28.84 \pm 2.03$ & 0.26 \\
ASA & & & \\
I & $17(65.7 \%)$ & $21(70.0 \%)$ & 0.422 \\
II & $13(43.3 \%)$ & $9(30.0 \%)$ & 0.869
\end{tabular}

Table 1: Comparison between the two studied groups according to demographic data

*: Statistically significant $(\mathrm{p}<0.05)$.

\begin{tabular}{cccc}
\hline & $\begin{array}{c}\text { Group TE } \\
(\mathbf{n = 3 0})\end{array}$ & $\begin{array}{c}\text { Group ES } \\
(\mathbf{n}=\mathbf{3 0})\end{array}$ & P-value \\
\hline Time to give the block & $14.1 \pm 2.1$ & $7.8 \pm 1.8$ & $<0.001^{*}$ \\
\hline
\end{tabular}

Table 2: Comparison between the two studied groups according to the time needed to give block

*: Statistically significant $(\mathrm{p}<0.05)$.

\begin{tabular}{cccc}
\hline $\begin{array}{c}\text { Mean arterial blood pressure } \\
(\mathbf{m m H g})\end{array}$ & $\begin{array}{c}\text { Group TE } \\
(\mathbf{n = 3 0})\end{array}$ & $\begin{array}{c}\text { Group ES } \\
(\mathbf{n}=\mathbf{3 0})\end{array}$ & P-value \\
\hline 0 & $68.2 \pm 3.6$ & $80.4 \pm 3.4$ & $<0.001^{*}$
\end{tabular}




$\begin{array}{cccc}60 \mathrm{~min} . & 68.1 \pm 2.2 & 68.5 \pm 3.2 & 0.61 \\ 120 \mathrm{~min} . & 68.0 \pm 2.2 & 67.6 \pm 3.3 & 0.52 \\ 3 \mathrm{hr} . & 67.7 \pm 2.5 & 68.0 \pm 3.1 & 0.64 \\ 4 \mathrm{hr} . & 68.1 \pm 2.6 & 68.7 \pm 3.5 & 0.41 \\ 6 \mathrm{hr} . & 67.4 \pm 1.8 & 68.3 \pm 2.5 & 0.12 \\ 12 \mathrm{hr} . & 67.8 \pm 2.0 & 68.4 \pm 2.8 & 0.37 \\ 24 \mathrm{hr} . & 67.6 \pm 2.7 & 68.5 \pm 2.9 & 0.21\end{array}$

Table 3: Comparison between the two studied groups according to Mean arterial blood pressure $(\mathrm{mm} \mathrm{Hg})$ *: Statistically significant $(\mathrm{p}<0.05)$.

\begin{tabular}{cccc}
\hline $\begin{array}{c}\text { VAS } \\
\text { (IQR-median) }\end{array}$ & $\begin{array}{c}\text { Group TE } \\
(\mathbf{n = 3 0})\end{array}$ & $\begin{array}{c}\text { Group ES } \\
(\mathbf{n}=\mathbf{3 0})\end{array}$ & P-value \\
\hline 0 & $1(0-1)$ & $0(0-1)$ & $<0.001^{*}$ \\
$2 \mathrm{hr}$. & $1(1-2)$ & $1(0-1)$ & $<0.001^{*}$ \\
$4 \mathrm{hr}$. & $2(1-2)$ & $1(1-1)$ & $<0.001^{*}$ \\
$6 \mathrm{hr}$. & $2(2-3)$ & $1(1-2)$ & $<0.001^{*}$ \\
$12 \mathrm{hr}$. & $3(2-4)$ & $2(1-3)$ & $<0.001^{*}$ \\
$24 \mathrm{hr}$. & $3(2-4)$ & $2(1-3)$ & $<0.001^{*}$ \\
\hline
\end{tabular}

Table 4: Comparison between the two studied groups according to visual analogue scale VAS: visual analogue scale, IQR: interquartile range, *: Statistically significant $(\mathrm{p}<0.05)$.

\begin{tabular}{cccc}
\hline Number of morphine boluses & $\begin{array}{c}\text { Group TE } \\
(\mathbf{n = 3 0})\end{array}$ & $\begin{array}{c}\text { Group ES } \\
(\mathbf{n}=\mathbf{3 0})\end{array}$ & P-value \\
\hline 0 & $9(30.0 \%)$ & $19(63.3 \%)$ & $0.01^{*}$ \\
1 & $6(20.0 \%)$ & $6(20.0 \%)$ & 1.00 \\
2 & $7(23.3 \%)$ & $4(13.3 \%)$ & 0.51 \\
3 & $8(27.0 \%)$ & $1(3.3 \%)$ & $0.01^{*}$
\end{tabular}

Table 5: Comparison between the two studied groups according to Number of morphine boluses *: Statistically significant $(\mathrm{p}<0.05)$.

\begin{tabular}{cccc}
\hline & $\begin{array}{c}\text { Group TE } \\
(\mathbf{n = 3 0 )}\end{array}$ & $\begin{array}{c}\text { Group ES } \\
(\mathbf{n = 3 0 )}\end{array}$ & P-value \\
\hline Patients satisfaction & & & 0.072 \\
0-3 (not satisfied & $11(36.7 \%)$ & $4(13.3 \%)$ & 0.779 \\
4-6 (partly satisfied) & $10(33.3 \%)$ & $8(26.7 \%)$ & $0.037 *$ \\
7-10 (highly satisfied) & $9(30.0 \%)$ & $18(60.0 \%)$ & 0.007 ) \\
\hline
\end{tabular}

Table 6: Comparison between the two studied groups according to Patients satisfaction

*: Statistically significant $(\mathrm{p}<0.05)$.

\section{DISCUSSION}

The need for ideal post-operative pain management is crucial. Although thoracic epidural analgesia (TEA) has been considered as the golden standard, Erector spinae plane (ESP) block is now emerging as a better alternative technique. The value of ESP block as a rescue analgesic technique was highlighted by Forero et al ${ }^{15}$ in a case report discussing thoracotomy after a failed epidural technique.

In the present study, the time needed to give the anaesthesia block, mean arterial blood pressure (MABP), visual analogue scale (VAS) assessment for post-operative pain, boluses of morphine, and patient satisfaction were evaluated. Main complications as hypotension were recorded.

The time needed in our study to give the anesthesia block was found to be significantly lower in group ES that received erector spinae plane (ESP) block $(\mathrm{p}<0.001)$ as compared to group TE. The pain score assessed in this work according to the visual analogue scale (VAS) was significantly better in group ES as compared to group TE $(p<0.001)$. Post-operative readings of MABP tended to be lower in the group (TE), but without statistically significant difference $(p>0.05)$. In support of our results, Nagaraja et al ${ }^{16}$, recorded VAS scores persistently $\leq 4$ until $48 \mathrm{~h}$ in 2 study groups of TEA and ESP block performed for post-thoracotomy pain until $12 \mathrm{~h}$ post-extubation. They stated that the VAS scores were comparable between both groups, with the relative advantage of ESP block to be easier to perform as compared to TEA and paravertebral block (PVB) in breast surgeries. Similarly, Chin et al ${ }^{17}$ stated that ESP block avoided the complications attributed to those two major methods, as they had negligible effects on the dorsal, ventral, or communicant rami of the spinal nerve roots. Furthermore, Bonvicini et al ${ }^{18}$ suggested that the use of ultrasound-guided (USG) ESP block in 
bilateral breast cancer reconstructive surgery can be an effective safe alternative to TEA and paravertebral block techniques.

The administered boluses of morphine recorded in our results were significantly lower in group ES in comparison to group TE patients, as 3.3\% of patients in group ES received 3 boluses compared to $27 \%$ ( $\mathrm{p}=0.01$ ). In accordance, Singh and colleagues ${ }^{10}$ documented that a significant decrease in the requirement of postoperative morphine in patients who received erector spinae plane block, and patient satisfaction scores were better in ESP group. Similarly, Gürkan and coworkers ${ }^{19}$ in a randomized control trial on the analgesic effect of US-guided single shot ESP for breast surgery observed a statistically significant decrease in postoperative morphine consumption, establishing its role for analgesia and postoperative opioid-sparing effect. Nair et $\mathrm{al}^{20}$ reported efficacy of this block in a case series of 5 patients subjected to thoracic surgery. Also, previous authors stated in their case reports that both visceral and somatic pain were efficiently abolished by the use of ESP block ${ }^{21,22}$.

In agreement, Kimachi et al ${ }^{23}$ used US-guided ESP for accomplishing surgical anesthesia for a right-sided mastectomy and axillary dissection in a patient with high cardiovascular risk, and she had a minimal requirement of postoperative analgesia. They explained that the ability to carry out the anesthesia was attributed to that ESP isn't a limited area surrounded by the spinal column as compared with the epidural zone. The ESP plane is larger than the epidural space as the erector spinae muscle runs along the length of the thoracolumbar spine, thus providing extensive craniocaudal spread ${ }^{24}$.

More patients were highly satisfied in this study in group ES $(60 \%)$ than group TE $(30 \%)(p=0.037)$. This coincides with Forteo et al ${ }^{9}$ who recommended the use of ESP block for patients with chronic thoracic neuropathic pain, who were poorly responsive to oral pharmacotherapy. The authors attributed this effect to the extent of the cutaneous sensory block when an injection of $25 \mathrm{ml}$ of LA was administered at the level of $\mathrm{T} 5$, as it spreads cephalocaudal over the anterior-posterior thorax ranging from $\mathrm{T} 1$ to $\mathrm{T} 11$.

\section{CONCLUSION}

Ultrasound-guided ESP block is a good choice with a better outcome in patients undergoing elective mastectomy as regards better efficiency, satisfaction, and fewer complications.

\section{REFERENCES}

1. Porzionato A, Macchi V, Stecco C, Loukas M, and Tubbs RS et al. Surgical anatomy of the pectoral nerves and the pectoral musculature. Clin Anat, 2012;25(5):559-75.
2. Bolin ED, Harvey NR, and Wilson SH. Regional Anesthesia for Breast Surgery: Techniques and Benefits. Curr Anesthesiol Rep, 2015; 5(2):217-24.

3. Macrae WA. Chronic post-surgical pain: 10 years on. Br J Anaesth, 2008;101(1):77-86.

4. Apfelbaum JL, Ashburn MA, Connis RT, Gan TJ and Nickinovich DG et al. Practice guidelines for acute pain management in the perioperative setting: An updated report by the American Society of Anesthesiologists Task Force on Acute Pain Management. Anesthesiology, 2012;116(2):248-73.

5. Nagaraja PS, Ragavendran S, Singh NG, Asai O and Bhavya $G$ et al. Comparison of continuous thoracic epidural analgesia with bilateral erector spinae plane block for perioperative pain management in cardiac surgery. Ann Card Anaesth, 2018;21(3):323-7.

6. Blanco R. The 'pecs block': a novel technique for providing analgesia after breast surgery. Anesthesia, 2011;66(9):847-8.

7. Wu CL, Naqibuddin M, Rowlingson AJ, Lietman SA and Jermyn RM et al. The effect of pain on health related quality of life in the immediate postoperative period. Anesth Analg, 2003; 97(4): 1078-85.

8. Dhole S, Mehta Y, Saxena H, Juneja R and Trehan N. Comparison of continuous thoracic epidural and paravertebral blocks for postoperative analgesia after minimally invasive direct coronary artery bypass surgery. $J$ Cardiothorac Vasc Anesth, 2001;15(3):288-92.

9. Forero M, Adhikary SD, Lopez H, Tsui C and Chin KJ. The erector spinae plane block: A Novel analgesic technique in thoracic neuropathic pain. Reg Anesth Pain Med. 2016;41(5):621-7.

10. Singh S, Kumar G and Akhileshwar. Ultrasoundguided erector spinae plane block for postoperative analgesia in modified radical mastectomy: A randomised control study. Indian $J$ Anaesth. 2019;63(3):200-4.

11. Ince I, Ozmen O, Aksoy M, Zeren S and Ulas AB et al. Erector spinae plane block catheter insertion under ultrasound guidance for thoracic surgery: Case Series of Three Patients. Eurasian J Med. 2018; 50(3): 204-6.

12. Hamilton DL and Manickam B. Erector spinae plane block for pain relief in rib fractures. Br J Anaesth, 2017;118(3): 474-5.

13. Von Baeyer CL, Bergstrom KJ, Brodwin MG, and Brodwin SK. Invalid use of pain drawings in 
psychological screening of back pain (TLIP) block: A pilot study in volunteers. Can J patients. Pain. 1983;16(1):103-7. Anaesth. 2015;62(11):1196-200.

14. Subramanian B, Shastri N, Aziz L, Gopinath R, and Karlekar A et al. ASSIST- Patient satisfaction survey in postoperative pain management from Indian subcontinent. $J$ Anaesthesiol Clin Pharmacol. 2017;33(1):40-7.

15. Forero M, Rajarathinam M, Adhikary S, and Chin KJ. Continuous erector spinae plane block for rescue analgesia in thoracotomy after epidural failure: A Case report. A Case Rep. 2017;8(10):254-6.

16. Nagaraja PS, Ragavendran S, Singh NG, Asai O and Bhavya $G$ et al. Comparison of continuous thoracic epidural analgesia with bilateral erector spinae plane block for perioperative pain management in cardiac surgery. Ann Card Anaesth. 2018;21(3):323-7.

17. Chin KJ, Malhas L, and Perlas A. The erector spinae plane block provides visceral abdominal analgesia in bariatric surgery: a report of 3 cases. Reg Anesth Pain Med. 2017; 42(3): 372-6.

18. Bonvicini D, Tagliapietra L, Giacomazzi A, and Pizzirani E. Bilateral ultrasound.guided erector spinae plane blocks in breast cancer and reconstruction surgery. J Clin Anesth. 2018;44:3-4.

19. Gürkan Y, Aksu C, Kuş A, Yörükoğlu UH and Kılıç CT. Ultrasound guided erector spinae plane block reduces postoperative opioid consumption following breast surgery: A randomized controlled study. J Clin Anesth. 2018;50:65-8.

20. Nair AS, Seelam S, Naik V, and Rayani BK. Opioid-.free mastectomy in combination with ultrasound - Guided erector spinae block: A series of five cases. Indian J Anaesth. 2018;62(8):632-4.

21. Bonvicini D, Giacomazzi A and Pizzirani E. Use of the ultrasound- guided erector spinae plane block in breast surgery. Minerva Anestesiol. 2017; 83(10): 1111 2.

22. Selvi O and Tulgar S. Use of the Ultrasound-Guided Erector Spinae Plane Block in Segmental Mastectomy. Turk J Anaesthesiol Reanim. 2019;47(2):158-60.

23. Kimachi PP, Martins EG, Peng P and Forero M. The erector spinae plane block provides complete surgical anesthesia in breast surgery: A case report. A Pract. 2018;11(7):186-8.

24. Hand WR, Taylor JM, Harvey NR, Epperson TI, and Gunselman RJ et al. Thoracolumbar interfascial plane 